\title{
Gas Sensor Array with Polymer-carbon Glue Composite Structures for Neural Network Application
}

\author{
Bo-Chun Yang and Yu-Jen Hsiao* \\ Department of Mechanical Engineering, Southern Taiwan University of Science and Technology, \\ Tainan City, 71005, Taiwan
}

(Received May 18, 2021; accepted December 6, 2021)

Keywords: gas sensor, sensor array, volatile organic compounds, neural network, stacked composite membranes

In this study, we focus on the gas sensor array with semiconductor and neural network applications. Two different sensing membranes, namely, diluted conductive water-soluble carbon glue and polymer-carbon glue stacked membranes, were used for experiments to compare the differences and correlations between multiple polymers and multiple gases. In the experiments, the reactions of 13 different polymers with five gases (air, ethanol, $\mathrm{CO}, \mathrm{NO}_{2}$, and $\mathrm{NH}_{3}$ ) were tested. A set of sixteen array sensors with a line width of $50 \mu \mathrm{m}$ and a sensing diameter of $3 \mathrm{~mm}$ were used, and such sensors were coated with a 300-nm-thick aluminum-doped zinc oxide (AZO) layer on the wafer surface. The gas sensitivity obtained using various polymers dripped on the sensing area was tested, and the sensitivity of small low-energy heterogeneous gassensing elements is discussed. It is concluded that the various gases considered have good separation properties and excellent selectivity and reproducibility. Among the two different sensing membranes, the gas reaction ratio of $(\Delta R / R)_{\max }=149 \%$ is the most prominent relative to the resistance of carbon glue in reference to the air baseline; as a result, the carbon membrane showed a higher gas sensitivity than the polymer-carbon membrane.

\section{Introduction}

In recent years, electronic nose systems have been miniaturized to reduce manufacturing costs and provide higher precision gas sensitivity and specificity than in the past decade. ${ }^{(1)}$ The sensing material triggers a physical reaction with an absorbed analyte and then changes its resistivity or dielectric constant. The concentration of the analyte can be determined from the property variation. ${ }^{(2)}$ During the detection process, gas sensors based on metal oxides will inevitably be cross-contaminated by interfering gases. It has been considered that by using sensor arrays with different characteristics, gas recognition results can be improved, which is a potential solution to this problem. ${ }^{(3,4)}$

Polymer-based sensing materials are usually fabricated in the form of membranes. In addition to excellent adaptability with microstructure sensors, membrane sensors can also enhance the 
adsorption and desorption capabilities of aerosols, improving sensor sensitivity and rapid reuse capabilities. ${ }^{(5)}$ The conductive carbon glue and polymer stacked sensor used in this study are simple to operate, and the solution drop-casting process can be used to easily control the quality of the sensing membrane. Carbon structural materials have unique physical properties, such as excellent corrosion resistance and outstanding electronic conductivity. These properties make them suitable for use as carriers for electrodes or electrode catalysts. Carbon materials, which are used with various gases, have been ideal for high-sensitivity gas sensors.

Depending on the different gases and their concentrations, sensors in the array will show various changes in resistance due to physical mechanisms. Sorting can select polymer sensing materials with varying sensing responses to different gases to enhance gas specificity. By principal component analysis (PCA) methods or using neural network classification algorithms, the analysis of data to determine concentration and gas specificity can be simplified. ${ }^{(6-8)}$ The analytes in this experiment include five gases (air, ethanol, $\mathrm{CO}, \mathrm{NO}_{2}$, and $\mathrm{NH}_{3}$ ) and two different types of membrane (diluted conductive water-soluble carbon glue and polymer-carbon glue stacked membranes). ${ }^{(9)}$

The classification accuracy and overall performance of neural classifiers will be evaluated and compared to find the best algorithm for accurate gas recognition. This method can also be applied to other polymer-based sensors to selectively identify gases in various mixtures and make concentration predictions.

\section{Experimental Procedure}

\subsection{Design and fabrication}

This device is an electronic nose application that can accurately improve the measurement results of the object to be measured. The sensor array consists of 16 gas sensing areas and 20 electrodes. It is composed of $5 \times 4$ arrays of independent sensing electrodes to facilitate the measurement of the resistance response of each sensing area. The chip size obtained after bonding with the circuit board is $30.5 \times 30.5 \mathrm{~mm}^{2}$.

Figure 1 shows the blueprint for the design of a 16-sensor array. After many modifications, we decided to use this array method to reduce the chip size significantly. By putting more membrane sensors in a particular area, the sensor chip size becomes $19.5 \times 19.5 \mathrm{~mm}^{2}$. Each circular membrane sensor is designed with interdigital electrodes with a line width of $50 \mu \mathrm{m}$, and the diameter is limited to $3 \mathrm{~mm}$ to minimize the heat loss of the silicon substrate.

The circuit board has 20 copper metal contacts (one negative and four positives on each side) for wire bonding and measurement with the chip body. The outer frame size is $30.5 \times 30.5 \mathrm{~mm}^{2}$, and the diameter of the contact point is set to $1 \mathrm{~mm}$.

The 6-inch wafers shown in Fig. 2 were fabricated by the Taiwan Semiconductor Research Center based in Southern Taiwan Science Park. The thickness of the silicon substrate was $400 \mu \mathrm{m}$. Membrane and sensor device samples were prepared for sputtering to deposit the aluminum-doped zinc oxide (AZO) structure; the thickness of AZO on top of the wafer surface was about $300 \mathrm{~nm}$. An AZO target (purity: 99.99\%) was used in the sputter system for 


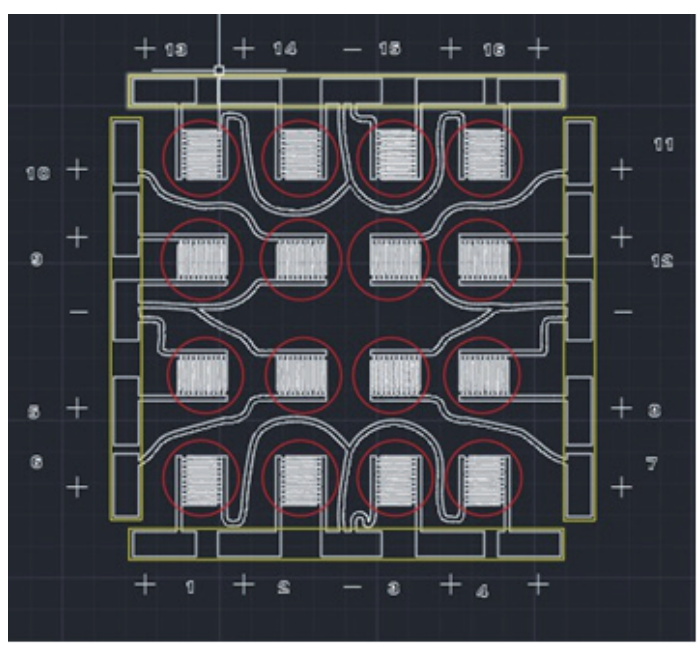

Fig. 1. (Color online) Schematic of sensor array arrangement.

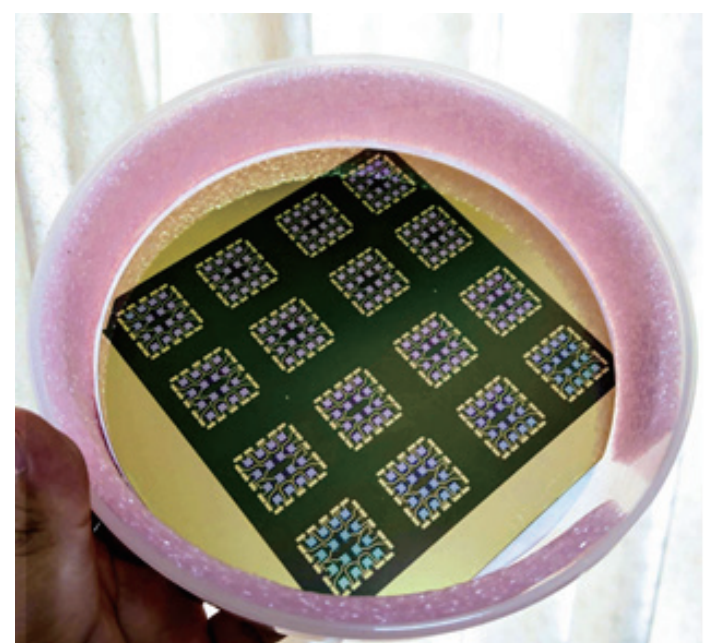

Fig. 2. (Color online) Sensors are arranged on the wafer and distributed into 16 chips.

deposition. The power supply to the sputtering gun in the deposition chamber was $80 \mathrm{~W}$, and the working pressure of the chamber was $10 \times 10^{-3}$ Torr. For sputter deposition, argon (Ar) and oxygen $\left(\mathrm{O}_{2}\right)$ gases were used in the chamber, and their flow rates were 30 and $1.5 \mathrm{sccm}$, respectively.

We used laser cutting to take out the chips from the wafer. Furthermore, the electrode is made of aluminum of $300 \mathrm{~nm}$ thickness. However, the melting of the aluminum electrode edge is caused by high temperature, resulting in resistance uncertainty, which will in turn result in sensing measurement deviations. Therefore, we presumed a space of $0.5 \mathrm{~mm}$ on each side of each chip, which was left to form a high-temperature buffer zone.

A wire bonding machine was used to bond the chip to the circuit board, and an aluminum wire was used as the conductive wire. However, because the electrode used in this wafer is also made of aluminum, the cohesiveness during wire bonding is ineffective. Later, we adjusted the parameters for several batches to increase the thickness of the aluminum electrode to $300-500 \mathrm{~nm}$. As a result, the cohesiveness between the electrode and the conductive wire was significantly improved.

\subsection{Experimental setting}

Figure 3 shows the resistance measurement of the chip performed using the Keithley PXI SMU system under an LED UV light source with an output power of $1 \mu \mathrm{A}$. The sensor array is placed in a custom-made transparent acrylic gas chamber (volume $18 \mathrm{~L}, 30 \times 30 \times 20 \mathrm{~cm}^{3}$ ).

In this experiment, we used five different target gases, namely, air, ethanol, $\mathrm{CO}, \mathrm{NO}_{2}$, and $\mathrm{NH}_{3}$, to establish a gas sensory database, and the net flow volume of the target gas (air + analyte) is fixed at $2 \mathrm{ml}$. The flow controller (FC) independently controls the flow of each gas to ensure that the gas will not suffer from cross-contamination. The PXI SMU system captures the sensor array resistance at a constant voltage. After the gas is exposed, the equipment can restore the 


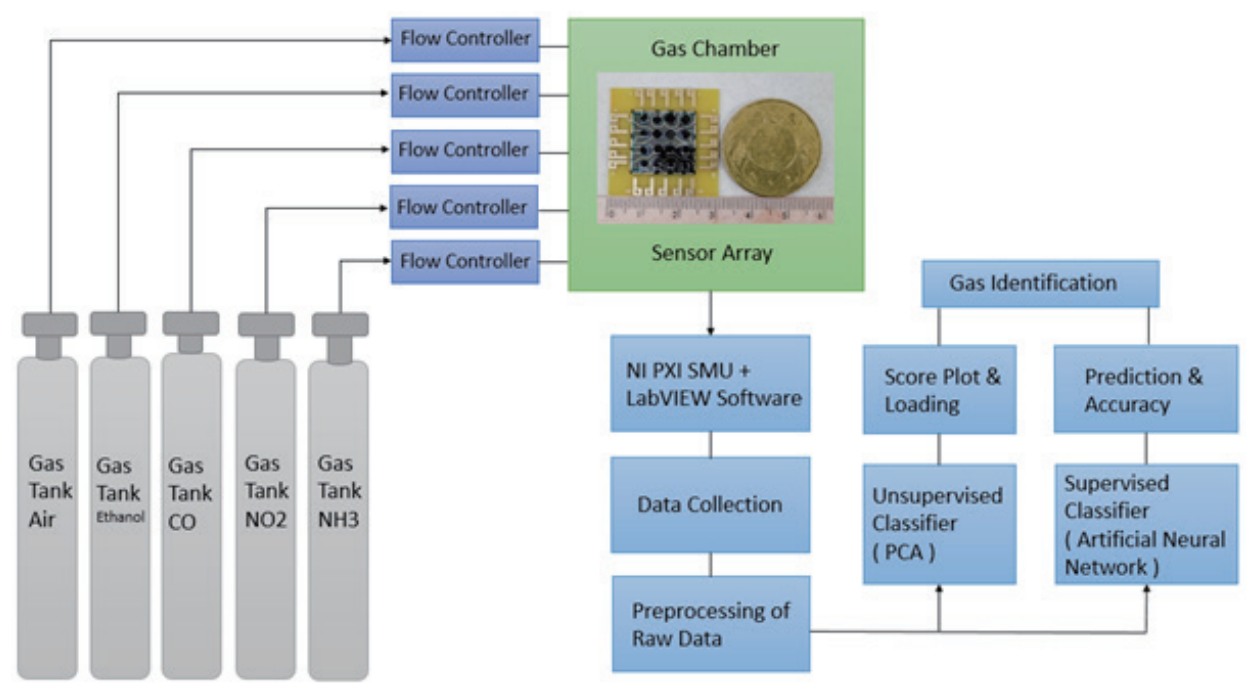

Fig. 3. (Color online) Gas sensing data collection and analysis.

baseline resistance without cleaning the gas chamber. After obtaining the original data, the gas response is mainly analyzed by two methods: unsupervised classification and supervised classification. PCA is an unsupervised method of feature extraction and recognition. The basic concept of PCA is to transform the original features into a set of new features in order of importance through a set of orthogonal vectors. These new features are linear combinations of the original functions and are independent of each other. The features extracted from the training data using PCA will be applied to the test data for gas recognition. Next, the training data are used to optimize different supervised classification algorithms covering a variety of neural networks. In the experiment, a fourfold cross-validation method is used to avoid the overfitting or distortion of the training data.

We placed the chip on a fixed platform and observed the internal condition through the transparent acrylic air chamber. In the corner of the cavity, we placed a microfan to accelerate the diffusion of the target gas to achieve a balanced state; at the contact point of the chip, 20 test pins were also preinstalled to measure resistance. Therefore, the resistance data of 16 circular membrane sensors can be collected in real time to avoid data errors, as shown in Fig. 4.

In this experiment, we also used 13 different functional polymer fluids and diluted conductive water-soluble carbon glue for the conductive film test in the sensing area. The testing modules were divided into two categories, namely, carbon glue and polymer-carbon glue.

Functional polymers with hydrogen bond acidity, alkalinity, and neutrality were used. Sol-gel was used as the process method. Thirteen types of polymer, namely, P (VDC-AN), SAA, PMVEMA, PVP, HPMC, PMS, PVBC, PEA, PVCMM, OV-210, PIP, PC, and PS, were used in this experiment and dripped on the sensing area to form the membranes.

The sensitivity and stability of the polymer sensing resistance variation can determine whether the swelling mechanism of the sensing film is stable. To enable the sensor to be used repeatedly in a short period, the sensing gas and the polymer sensing film are generally reversible, so most will use physical adsorption. Various polymer structures were used as sensing materials in the experiment. 


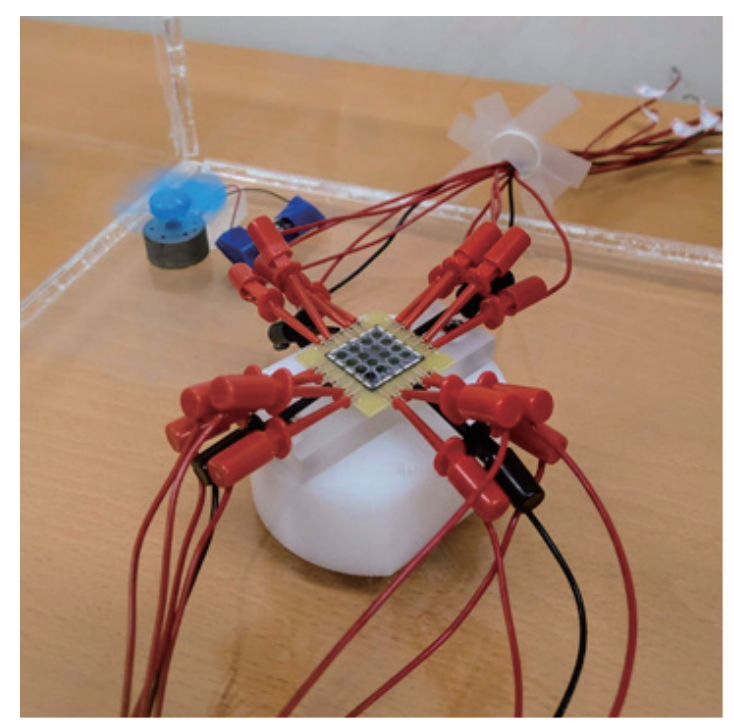

Fig. 4. (Color online) The sensor chip is used for measurement in the gas chamber.

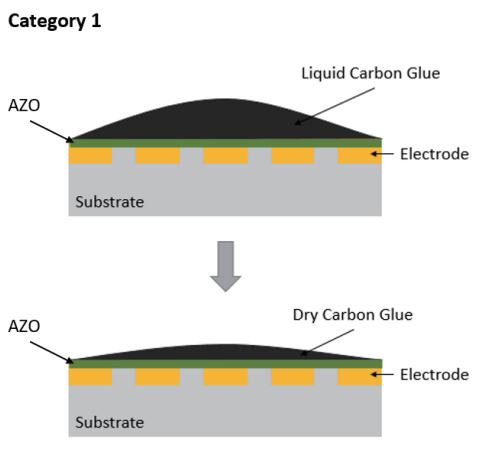

(a)

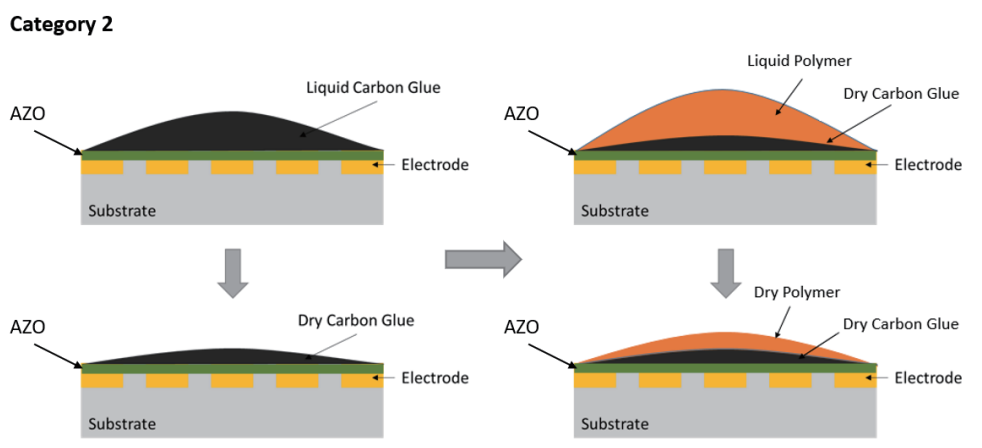

(b)

Fig. 5. (Color online) Two different drop-casting processes to form the sensing membranes. (a) Carbon membrane and (b) polymer-carbon membrane.

Because the chip body has 16 circular sensors, the carbon glue part (category 1) depicted in Fig. 5 was individually dripped on top of the 16 circular sensing areas. In the other part of the polymer-carbon glue (category 2) depicted in Fig. 5, the carbon glue was dripped on the surface of the sensing area first, and then the polymer liquid was also dripped on the sensing area of the electrodes numbered 1-13 in sequence. The 13 different types of polymer liquid were used for electrodes 1-13. For the remaining electrodes (electrodes 14-16), the polymer for the 13th electrode was used. The droplet volume of all the above fluids was $0.2 \mu \mathrm{L}$.

We used two different solution drop-casting methods to fabricate 16 membranes on the sensor chip. Figure 5 shows the processing steps. The drying was carried out in a vacuum oven heated to $50^{\circ} \mathrm{C}$, and the sensor chip was placed in a vacuum to dry for $1 \mathrm{~h}$. A moderate vacuum pressure can ensure that aerosols will not adsorb on the polymer surface during high-temperature drying. ${ }^{(10-12)}$ 


\section{Results and Discussion}

The sensor response to gas is generally considered a phase-time response, as shown in Fig. 6. The first step of odor analysis is to measure the reference gas, i.e., dry air, through the sensor to obtain the resistance baseline. Then, exposing the sensor to the target gas will cause its output signal to change until the resistance reaches a steady state. Then, the gas supply is turned off and the chamber is flushed with dry air to obtain another benchmark. The above test process is repeated several times to obtain the required sensing response data.

The typical sensing curve of one cycle is shown in Fig. 6 . The periods $t_{1}, t_{2}$, and $t_{3}$ correspond to the test times of flushing with dry air, test gas, and dry air, respectively. $t_{3}$ is also called the recovery time. The vertical axis of the sensing curve represents the resistance $R$ of the sensor when gas is present, where $R_{b}$ and $R_{\max }$ represent the reference and maximum resistances of the sensor exposed to air and test gas, respectively.

The gas reaction can be determined from the relationship between the resistance variation $(\Delta R / R)$ and the test time, where $\Delta R / R=\left(R-R_{b}\right) / R_{b}$. The maximum $\Delta R / R$ on the curve is an indicator of the sensitivity of the sensing device, where $R_{b}$ is the resistance baseline under fresh air existing in the environment and $R_{\max }$ is the maximum resistance of the sensor exposed to air and test gas. Equation (1) is a typical equation used to calculate the gas sensing response of the sensor.

$$
(\Delta R / R)_{\max }=\left(R_{\max }-R_{b}\right) / R_{b}
$$

The diffusivity equation shown as Eq. (2) considers the effects of temperature and pressure on gas volume. In other words, the volume is standardized to the conditions of $25{ }^{\circ} \mathrm{C}$ and $760 \mathrm{~mm} \mathrm{Hg}$ (1013.2 MBar).

$$
r=2.216 \times 10^{6}\left(\frac{D M w P A}{T L}\right) \log \left(\frac{P}{P-p}\right)
$$

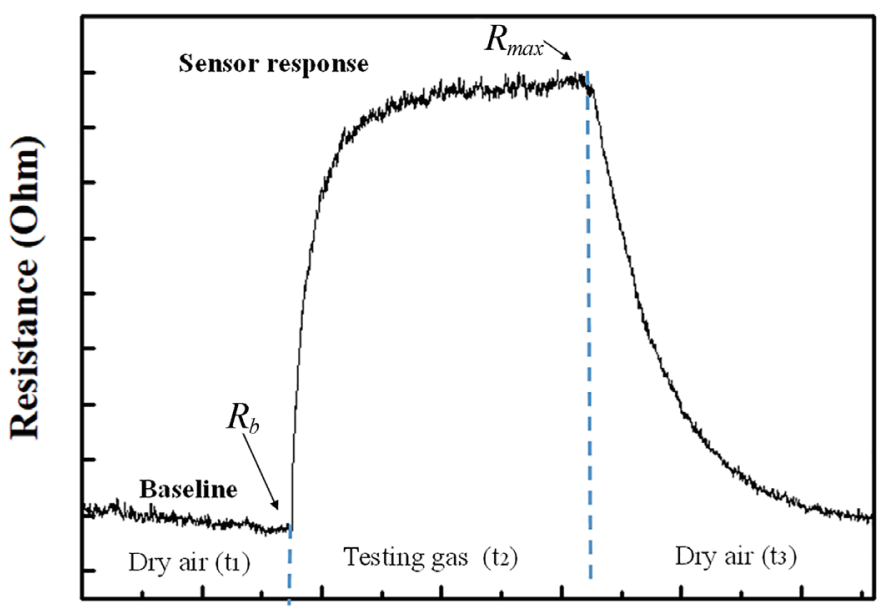

Time (s)

Fig. 6. (Color online) Typical gas sensing response curve. 
$r$ : diffusion rate, $\mathrm{ng} / \mathrm{min}$

$D$ : diffusion coefficient when the temperature is $T$ and pressure is $P, \mathrm{~cm}^{2} / \mathrm{s}$

$M w$ : molecular weight

$P$ : total pressure, Torr $\mathrm{Hg}$

$A$ : cross-sectional area of diffusion tube, $\mathrm{cm}^{2}$

T: temperature, $\mathrm{K}$

$L$ : diffusion tube length, $\mathrm{cm}$

$p$ : partial solvent pressure

We use the diffusivity to calculate the gas concentration of the dynamic carrier flow using Eq. (3). The concentration $C$ is expressed in ppm by volume, $r$ is the diffusion rate expressed in $\mathrm{ng} / \mathrm{min}$, and $F_{C}$ is the total flow rate of the mixed gas expressed in cc/min.

$$
C=(r \times K) / F_{C}
$$

Equation (4) is used to calculate the constant $K$ used in Eq. (3). $M w$ is the molecular weight of the pollutant gas, $22.4 \mathrm{~L}$ is one gram-mole of gas under reference conditions, which are $0{ }^{\circ} \mathrm{C}$ and $760 \mathrm{mmHg}, T$ is the room temperature expressed in ${ }^{\circ} \mathrm{C}$, and $P$ is the pressure expressed in $\mathrm{mm}$ Hg.

$$
K=\{22.4(T+273) \times 760\} / M w \times 273 \times P
$$

A neural network is a hierarchical machine learning method that gradually turns many disordered signals into helpful information and solves problems through layered processing. It contains many neurons, some of which are responsible for receiving and transmitting data. The neural network used in this experiment includes four layers of neurons. In addition to the input and output layers, there are two hidden layers in the middle responsible for delivering and processing data. These two hidden layers are internal layers that are not involved with input and output units.

According to the literature, a simple neural network with a single hidden layer can achieve an accuracy of more than $97 \%$. If it is improved, it can even reach an accuracy of more than $99 \%$. $(13,14)$ When learning objects are large and complex, a more significant framework scale is required, indicating that more neurons or hidden layers in the network are needed. Still, it does not necessarily mean that a deeper network is required. For the same 100 neurons, we can divide the framework into 1 layer of 100 neurons or 10 layers of 10 neurons. ${ }^{(15)}$ In a speech recognition test, whether with a shallow neural network such as a monolayer or a deep neural network with more than a single layer, the recognition rate increases with the number of neurons. However, with the same quantity of neurons, the performance of a deep neural network is always higher, so it is widely used.

Figure 7 shows that we provided six types of different learning benchmark data on the input layer in this process, namely, ethanol, $\mathrm{CO}, \mathrm{NO}_{2}, \mathrm{NH}_{3}$, temperature, and humidity. By calculating two layers of neurons, the main characteristics of the resistance data were captured in the first 


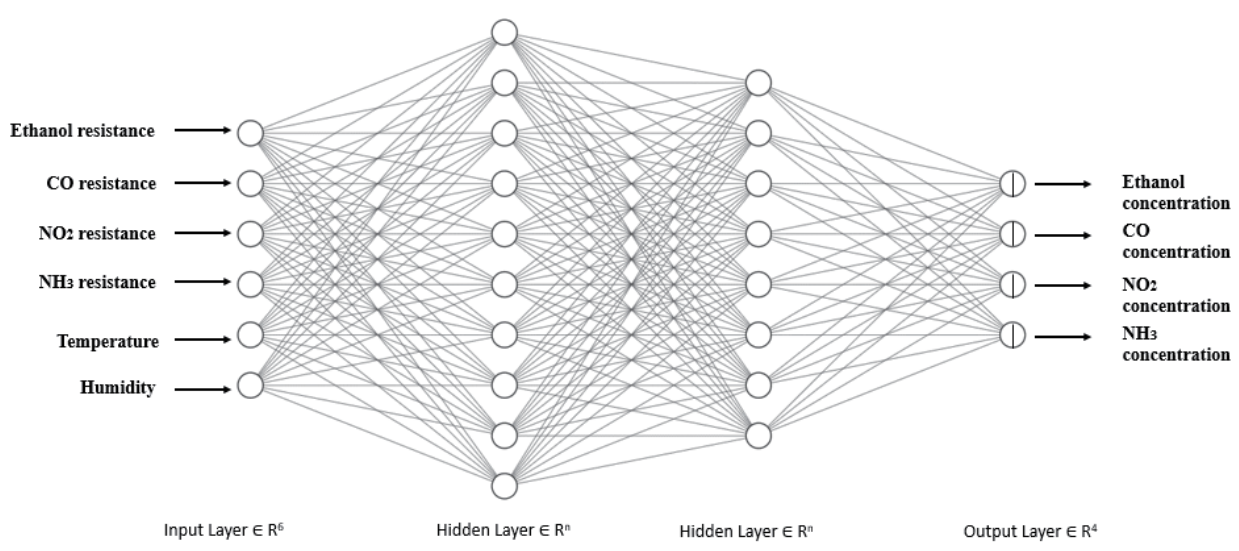

Fig. 7. Multiple gases and neural network architecture.

Table 1

Resistance difference of membrane sensor to carbon glue and polymer-carbon glue (unit: K-ohm).

\begin{tabular}{cccccc}
\hline Sensor & Air & Carbon & Ethanol & Polymer-carbon & Ethanol \\
\hline 1 & 483 & 503 & 469 & 70.2 & 370 \\
2 & 0.00539 & 0.03 & 0.02 & 0.00534 & 0.00537 \\
3 & 69.9 & 71 & 66 & 57.9 & 55.2 \\
4 & 4.965 & 9.14 & 9.13 & 5.518 & 5.4 \\
5 & 1000 & 880 & 850 & 790 & 910 \\
6 & 128.5 & 1270 & 1240 & 1220 & 1290 \\
7 & 14.75 & 9 & 11 & 37.9 & 37.12 \\
8 & 28.5 & 26.4 & 26.1 & 28 & 30.3 \\
9 & 70.3 & 850 & 810 & 320 & 810 \\
10 & 525 & 1310 & 1290 & 750 & 1290 \\
11 & 43.9 & 68 & 68 & 51.7 & 49.9 \\
12 & 58.4 & 13 & 10 & 24.8 & 17.4 \\
13 & 8.73 & -32.6 & -33.8 & 8.8 & 8.7 \\
14 & 3.71 & -1.3 & -1.4 & 3.7 & 3.73 \\
15 & 47.8 & 165 & 160 & 48.6 & 210 \\
16 & 9.6 & -29.3 & -29 & 9.77 & 9.72 \\
\hline
\end{tabular}

hidden layer. Analysis and filtering were performed on the second hidden layer, the various variables in the environment were considered, and finally, the concentration data of four gases were obtained in the output layer.

In fitting the experimental data, we collected the data measured by the sensor array and disassembled them into two parts; the first part is to read the resistance signal and then convert it into a gas reaction value through an equation. The second part is to collect environmental data, such as humidity and temperature, and normalize the two sources, then build a learning model. ${ }^{(16)}$ Finally, the test gases ethanol, $\mathrm{CO}, \mathrm{NO}_{2}$, and $\mathrm{NH}_{3}$ are injected into the chamber to capture the required data mentioned above; after that, the obtained data are input to the neural network; then, the model is compared with the previous experimental data to produce the final analysis result.

Table 1 shows the difference in the resistance of the sensor for the two surface droplets. First, we used the wafer without any coating to measure the resistance in dry air, obtain a baseline of 
resistance, and then measure the resistance after the carbon glue is dripped on the top surface. To obtain the resistance response, we injected ethanol into the wafer to observe the resistance variation. Finally, we added a polymer liquid on the surface of the carbon glue and injected ethanol for resistance measurement to compare the difference between the two.

From Fig. 8, it is found that the resistance of the chip added with carbon glue and polymer increased significantly. The amplitudes of the curves were fairly similar between the carbon membrane and the polymer-carbon membrane, and the carbon sample sensor showed a higher reproducibility than the polymer-carbon sensor sample.

In Table 2, four different gases are selected, namely, air, $\mathrm{CO}, \mathrm{NO}_{2}$, and $\mathrm{NH}_{3}$. From the above-mentioned 1 to 16 sensors, six sensors $(1,5,9,10,12$, and 15) were randomly selected for the selectivity test. These selected sensors were tested with the four different gases mentioned earlier. The selectivity test is conducted four times to determine if the sensor chip's selectivity to the target gas is effective. The dotted scatter diagrams shown in Fig. 9 depict that the membrane

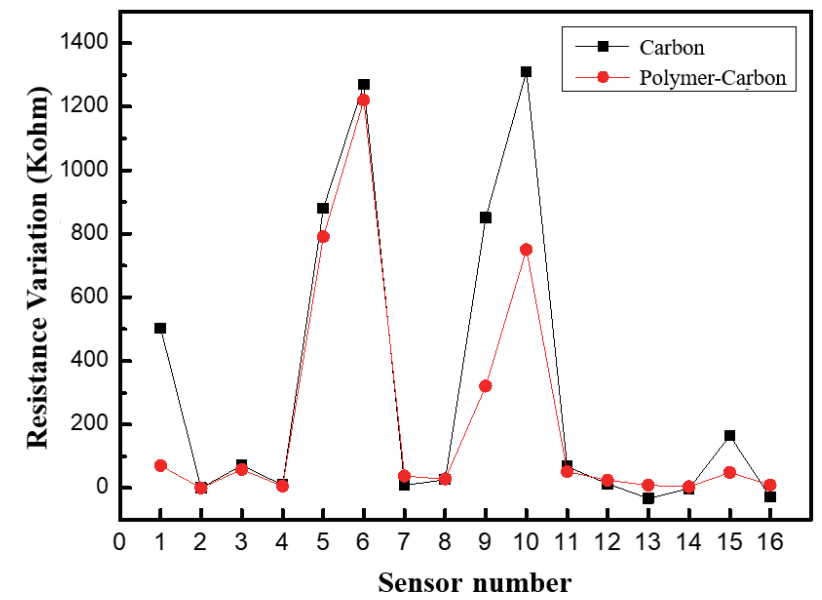

Fig. 8. (Color online) Resistance variations by carbon and polymer-carbon membranes.

Table 2

Randomly selected membrane sensors for gas selectivity testing (unit: K-ohm).

\begin{tabular}{cccccccccc}
\hline Selectivity 1 & Air & $\mathrm{CO}$ & $\mathrm{NO}_{2}$ & $\mathrm{NH}_{3}$ & Selectivity 3 & Air & $\mathrm{CO}$ & $\mathrm{NO}_{2}$ & $\mathrm{NH}_{3}$ \\
\hline 1 & 51.4 & 52.9 & 36.3 & 36.9 & 1 & 39.3 & 39.9 & 36.5 & 37.1 \\
5 & 243 & 245 & 233 & 243 & 5 & 260 & 266 & 210 & 212 \\
9 & 217 & 136.9 & 135.9 & 134.5 & 9 & 131.5 & 133.9 & 122.5 & 123.9 \\
10 & 590 & 540 & 510 & 490 & 10 & 480 & 440 & 364 & 385 \\
12 & 71.3 & 52.6 & 63.7 & 60.2 & 12 & 44.1 & 44.5 & 44.2 & 46.5 \\
15 & 32.9 & 29.9 & 25.1 & 25.7 & 15 & 21.9 & 22.5 & 22.9 & 21.8 \\
\hline Selectivity 2 & Air & $\mathrm{CO}$ & $\mathrm{NO}_{2}$ & $\mathrm{NH}_{3}$ & Selectivity 4 & Air & $\mathrm{CO}$ & $\mathrm{NO}_{2}$ & $\mathrm{NH}_{3}$ \\
\hline 1 & 35.3 & 37.8 & 36.2 & 34.9 & 1 & 34.1 & 33.7 & 31.2 & 33.6 \\
5 & 250 & 255 & 260 & 268 & 5 & 230 & 146.7 & 150.7 & 122.2 \\
9 & 142.3 & 138.9 & 134.5 & 135.5 & 9 & 127.5 & 132.8 & 123.9 & 100.3 \\
10 & 448 & 459 & 449 & 440 & 10 & 415 & 423 & 384 & 382 \\
12 & 61 & 57.6 & 58.5 & 62.5 & 12 & 41.3 & 42.9 & 41.3 & 42.5 \\
15 & 23 & 23.6 & 22.6 & 23 & 15 & 8.8 & 9.07 & 8.8 & 8.08 \\
\hline
\end{tabular}



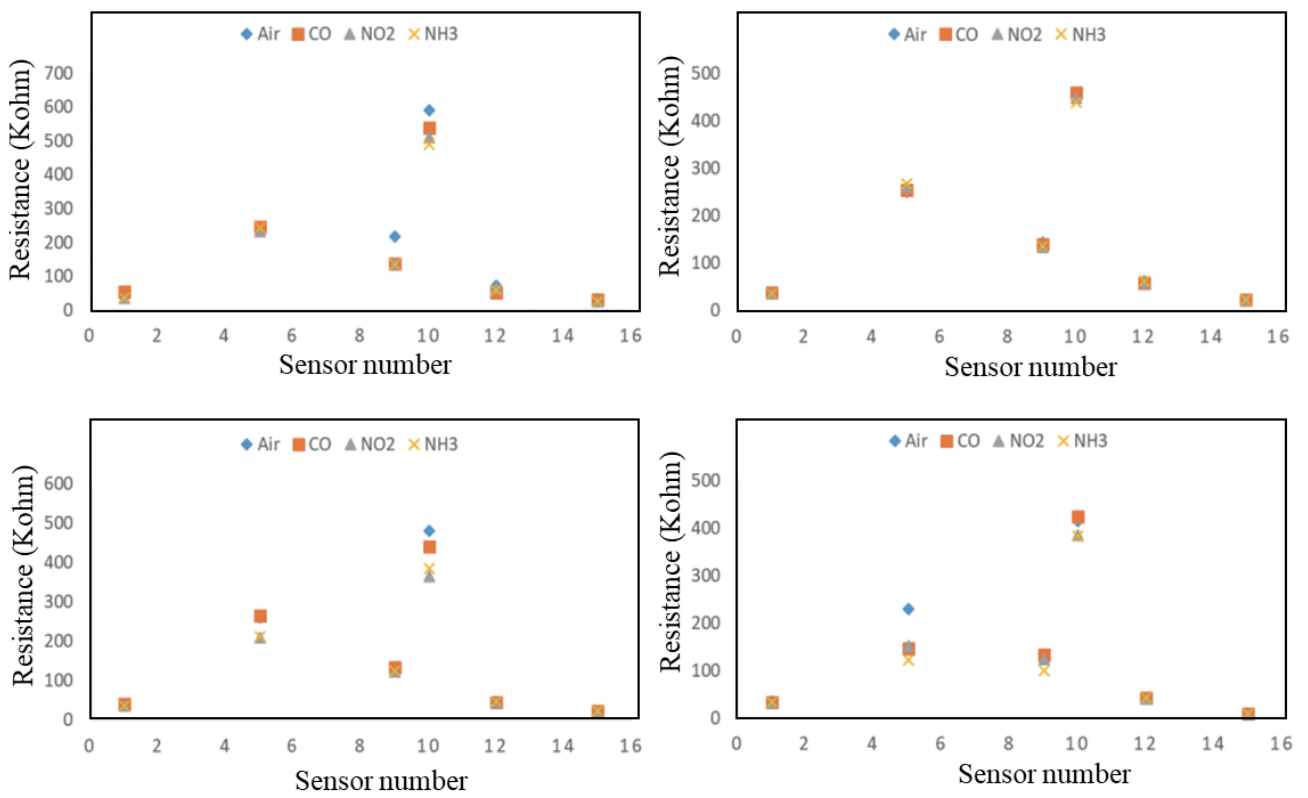

Fig. 9. (Color online) Selection and memory scatter diagram obtained using target gas.

sensors have specific selectivity and memory for the target gas in this experiment. After repeated tests, the point distributions are shared fairly similarly, indicating that the sensor chip is highly reliable and repeatable to distinguish gases, and shows excellent adaptability with a neural network.

\section{Conclusions}

In summary, a combination method was used to fabricate array sensors with 13 different functional polymers and carbon glue, and then neural networks were used to identify gases selectively. Five target gases can be placed and have outstanding reproducibility by only using a set of array sensors. A PCA study was carried out on the array reaction to detect air, ethanol, $\mathrm{CO}, \mathrm{NO}_{2}$, and $\mathrm{NH}_{3}$ gases, and the results showed a clear separation between the gas families. Next, a neural network-like network was used to train and optimize essential parameters. The method used shows excellent selectivity for specific gases in multiple sets of mixtures to screen out the best algorithm. It also proved that the solution drop-casting method's easy controllability can simplify the production of sensor membranes. As a result, the most prominent gas sensitivity can reach $(\Delta R / R)_{\max }=149 \%$ at room temperature and atmospheric humidity.

\section{Acknowledgments}

This work was financially supported by the Ministry of Science and Technology of Taiwan (project number MOST 110-2221-E-218-009). The authors also thank the Taiwan Semiconductor Research Institute for providing the wafer and sensor chip fabrication equipment and Kun-Han Tsai, Yu-Xuan Deng, and Huei-Wun Lyu for their assistance during the experiments. 


\section{References}

1 T. Julian, S. N. Hidayat, A. Rianjanu, A. B. Dharmawan, H. S. Wasisto, and K. Triyana: ACS Omega. 5 (2020) 29492. https://doi.org/10.1021/acsomega.0c04433

2 S. J. Young, Z. D. Lin: Microsyst. Technol. 24 (2018) 4207. https://doi.org/10.1007/s00542-018-3712-x

3 E. Gambi, G. Temperini, R. Galassi, and L. Senigagliesi: IEEE Sens. J. 20 (2020) 13562. https://doi.org/10.1109/ JSEN.2020.3005642

4 R. A. Potyrailo, J. Brewer, B. Cheng, M. A Carpenter, N. Houlihan, and A. Kolmakov: Faraday Discuss. 223 (2020) 161. https://doi.org/10.1039/D0FD00035C

5 T. Yoshizumi, T. Goda, A. Matsumoto, and Y. Miyahara: Sens. Mater. 30 (2018) 1001. https://doi.org/10.18494/ SAM.2018.1808

6 M. A. H. Khan, B. Thomson, R. Debnath, A. Motayed, and M. V. Rao: IEEE Sens. J. 20 (2020) 6020. https:// doi.org/10.1109/JSEN.2020.2972542

7 Z. Chen, Y. Zheng, K. Chen, H. Li, and J. Jian: IEEE Sens. J. 17 (2017) 1884. https://doi.org/10.1109/ JSEN.2017.2653400

8 X. Zhang, K. Zhang, D. Lin, Y. Zhu, C. Chen, L. He, X. Guo, K. Chen, R. Wang, Z. Liu, X. Wu, E. Long, K. Huang, Z. He, X. Liu, and H. Lin: Gigascience. 9 (2020) giaa011. https://doi.org/10.1093/gigascience/giaa011

9 B. Wang, J. C. Cancilla, J. S. Torrecilla, and H. Haick: Nano. Lett. 14 (2014) 933. https://doi.org/10.1021/ n1404335p

10 A. D. K. T. Lam, Z. D. Lin, H. Y. Lu, and S. J. Young: Microsyst. Technol. 28 (2019) 209. https://doi.org/10.1007/ s00542-019-04573-2

11 S. J. Young, Z. D. Lin: Microsyst. Technol. 24 (2018) 4207. https://doi.org/10.1007/s00542-018-3712-x

12 L. M. Wei, H. Y. Chen, J. Wang, W. E. Yuang, J. Zhao, D. Xu, and Y. F. Zhang: Sens. Mater. 26 (2014) 9. https:// myukk.org/SM2017/sm_pdf/SM965.pdf

13 M. Nielsen: Neural Networks and Deep Learning, Eds. (Determination press, San Francisco, CA, 2015) 1st ed., Chap. 1. https://static.latexstudio.net/article/2018/0912/neuralnetworksanddeeplearning.pdf

14 O. Russakovsky, J. Deng, H. Su, J. Krause, S. Satheesh, S. Ma, Z. Huang, A. Karpathy, A. Khosla, M. Bernstein, A. C. Berg, and L. Fei-Fei: Int. J. Comput. Vis. 115 (2015) 211. https://doi.org/10.1007/s11263-0150816-y

15 D. Yu, F. Seide, and G. Li: Proc. 2012 Int. Conf. Machine Learning (ICML, 2012). https://www.microsoft.com/ en-us/research/wp-content/uploads/2016/02/CD-DNN-HMM-ICML2012-Invited.pdf

16 V. V. Krivetskiy, M. D. Andreev, A. O. Efitorov, and A. M. Gaskov: Sens. Actuators, B 329 (2021) 129187. https://doi.org/10.1016/j.snb.2020.129187 